\title{
THE CHEMOTHERAPY OF NON-TUBERCULOUS INFECTIONS OF THE URINARY TRACT
}

By F. R. Kilpatrick, M.S., F.R.C.S., and Kenneth Maclean, M.D., M.R.C.P. Guy's Hospital

In the pre-antibiotic days the diagnosis of cystitis or pyelitis, confirmed by the finding of turbid urine containing pus cells, was considered adequate. The identification of the responsible organism was seldom of practical importance in deciding the line of treatment to be followed, and therapy consisted in administering large amounts of fluids and sufficient quantities of soda bicarbonate and potassium citrate to render the urine alkaline. If these measures were not effective it was sometimes found that sudden changes in the urinary $\mathrm{pH}$ might be successful and mandelic acid was given for this purpose. Today, surrounded as we are by a formidable battery of antibiotics, it is salutary to reflect that these old remedies, together with the natural resistance of the patient, were successful in eliminating the great majority of uncomplicated urinary infections and to remember that the principles they embody are still applicable whether or not an antibiotic is employed.

It is essential to realise that some acute and nearly all chronic urinary infections are primarily due to mechanical obstruction with resulting stasis. Such a state of affairs may be due to stone, growth or stricture, and failure to recognize this important fact is responsible for many of the cases not responding when an apparently efficient antibiotic is employed. Full urinary investigation should therefore be carried out in all cases of chronic or recurrent infection and in any who fail to show the expected response to treatment. A plain radiograph of the urinary tract, an intravenous pyelogram, cystoscopy and, if indicated, retrograde pyelography should be carried out, in addition to full microscopical and bacteriological investigation of the urine. If mechanical obstruction is found it is very unlikely that antibiotic therapy has more to offer than a temporary relief of symptoms and the indications for surgery must be considered on their merits.

Before considering the rôle of antibiotics in the control of urinary infections, it is necessary to discuss briefly the main advantages and dis- advantages of those in common use today. The 3 . sulphonamides, penicillin, streptomycin, aureomycin and chloramphenicol will be considered, $\dot{\omega}$ while reference will be made to two other sub- $\vec{\omega}$ stances at present difficult to procure in this ${ }_{\omega}^{\circ}$ country, namely terramycin and polymyxin $\mathrm{E}$.

\section{Sulphonamides}

Since Domagk in 1935 reported that prontosil $\frac{\Phi}{O}$ rubrum was therapeutically active in controlling streptococcal infections in mice, the sulphonamides have assumed a great importance in the contrgl $\vec{c}$ of infectious disease. They are active againsting large number of both gram positive and gra negative organisms, among the more importa in causing urinary infection being the streptococcus, the gonococcus and many strains of bacillus coli. They are very cheap and, if properly used, the risk attached to their administration is slight 0 indeed. The sulphonamides are concentrated $\overrightarrow{\overrightarrow{0}}$ by the kidneys in their passage from blood to 3 urine and it is thus unnecessary to give the large doses required for disease in other situations. With the exception of sulphanilamide, which is 음 therapeutically unsatisfactory in view of its many other toxic effects, all sulphonamides have a $\frac{5}{3}$ tendency to crystallize out in their passage through the renal tract. This most commonly occurs in $\frac{0}{3}$ the ureters; although occasionally the collecting tubules in the kidney may be affected. Haematuria, oliguria and eventually anuria will result. This $\frac{7}{0}$ complication is much more likely with some of the older less-soluble preparations and if the $N$ reaction of the urine is acid. When giving sul- $N$ phonamides it is therefore wise to insist on a fluid intake of at least five pints per day and if the $\omega$ patient is in hospital an 'intake and output ' chart should always be kept. The urine should be made alkaline to litmus by the simultaneous $\Phi$ administration of a mixture containing soda bicarbonate and potassium citrate in equal parts. If these precautions are observed and one of the $\frac{\overrightarrow{\mathbb{D}}}{\mathbb{0}}$ more soluble preparations such as sulphadimidine $\frac{?}{\mathbb{D}}$ 
(sulphamerazine) or sulphatriad used, the risk is negligible. It is nevertheless wise to avoid sulphonamide administration to patients with seriously impaired renal function. A 'loading' dose of $2 \mathrm{gm}$. followed by $0.5 \mathrm{gm}$. six-hourly is adequate and administration should be continued for six days.

Should oliguria or anuria occur, its treatment consists in increasing the intake of fluids orally and, if necessary, parentally. At the same time alkalis should be given and, if the anuria continues for eighteen hours under this regime, one or two pints of 3.8 per cent. sodium sulphate should be administered intravenously. Further prolongation of this state calls for the passage of a ureteric catheter on each side. It will be noted that this treatment differs from that advised when anuria develops in conjunction with a high blood urea (Bull, Joekes and Lowe, 1949). Occasionally, if the measures described above are not successful in restoring a satisfactory urinary output, a state of affairs especially likely to occur when the kidney function was initially poor, it may be necessary to abandon any attempt to flush the kidney and concentrate on the prevention of a dangerous azotemia developing before a spontaneous recovery of renal function has a chance to occur.

Other toxic effects are rare and occur mostly in people who have become sensitized to the drugs. Pyrexia and rashes of a macular type may occur at any time during treatment, and call for the withdrawal of the drug. Very rarely agranulocytosis may be seen, but this serious complication only occurs with prolonged or repeated administration: if it is suspected the sulphonamides must be stopped immediately, penicillin administered and further measures such as blood transfusion or the administration of pentnucleotide considered.

\section{Penicillin}

The common organisms found in the urinary tract which are sensitive to penicillin are the streptococcus, gonococcus, staphylococcus albus and certain strains of staphylococcus aureus. About 60 per cent. of the penicillin injected is excreted in the urine, so that moderate doses result in a high urinary concentration, and organisms comparatively resistant to penicillin may be attacked with success; 300,000 units of procaine penicillin twice daily should suffice to control most urinary infections due to sensitive organisms.

Penicillin is extremely useful in controlling the urethral and prostatic infections of sulphonamideresistant gonorrhoea and also in removing the residual staphylococcus albus infection so often found after the removal of renal calculi, thus diminishing the likelihood of relapse.

If used in the treatment of perinephric abscess $\stackrel{\unrhd}{\complement}$ (or renal carbuncle), 75 per cent. of which are $\stackrel{c}{\Rightarrow}$ due to the staphylococcus aureus, it may bring $\overrightarrow{\vec{s}}$ about complete resolution if given early. Here, $\overrightarrow{0}$ however, the main focus of infection is outside the urinary tract and heavier dosages than those $\frac{}{\omega}$ mentioned above should be used.

Rare toxic manifestations of penicillin are fever, angioneurotic oedema and various skin eruptions usually of an urticarial nature. It may be possible to control these by the administration of an antihistamine preparation such as Anthisan roo mgm. t.d.s., and they do not necessarily call for the immediate withdrawal of the penicillin. The antihistamine drugs are not very helpful for skin eruptions arising after the cessation of treatment.

\section{Streptomycin}

Streptomycin has a far wider sphere of action than penicillin, for not only does it attack most of the organisms mentioned above against which penicillin is active, but in addition many types of B. coli and some strains of B. proteus and Ps. pyocyaneus. Indeed, Fairbrother, Martyn and Parker (I95I) report that it is the most successfug of all antibiotics against the Ps. pyocyaneus, 3 per cent. of $5^{6}$ strains tested being sensitive. It not absorbed from the gastro-intestinal tract in satisfactory concentration and so must be given by injection. It is excreted by the kidneys and a daily amount of $2 \mathrm{gm}$. in two equally divided doses gives a satisfactory concentration in the urine. It has, however, one very serious disadvantage. Bacteria acquire resistance in a very short space of time, and such resistance is of high degree and permanent. Generally speaking, it is true to say that with the exception of the tubercle bacillus any organisms that have not been eradicated by streptomycin at the end of five days have acquired such a degree of resistance that further administration is useless. Second courses can therefore never be successful. In these circumstances it is probably wise to limit the use of streptomycin to those cases where bacteriological tests upon the sensitivity of the organism concerned show that it is the most suitable antibiotic available. It should also be remembered that it is very unlikely to be successful in the presence of urinary stasis and every effort should be made to remove any obstruction before it is given. An alkaline urine is said to enhance the effect of the drug.

Toxic reactions such as pyrexia and skin rashes are very rare. The more common manifestations of tinnitus, vertigo and deafness are almost unknown when the administration is continued for only five days. 


\section{Aureomycin and Chloramphenicol}

The action of these two antibiotics is extremely similar and they may best be discussed together. Aureomycin is derived from the 'streptomyces aureofaciens,' while chloramphenicol, originally obtained from the 'streptomyces venezuelas' under the name 'chloromycetin,' can now be produced synthetically. Both have a bacteriostatic action against a very large variety of organisms, the one important difference between them being in the salmonella group where chloramphenicol alone is active.

Their action is variable against different strains of B. coli, staphylococcus albus, B. subtilis, B. proteus and Ps. pyocyaneus, being especially disappointing against the latter four.

Aureomycin and chloramphenicol are absorbed incompletely from the intestinal tract and are both excreted in the urine, aureomycin to a very much greater degree than chloramphenicol. They are administered by the oral route in $250-\mathrm{mgm}$. capsules and a week's course of $500 \mathrm{mgm}$. sixhourly is adequate to deal with most urinary infections. The problem of bacterial resistance has not yet assumed serious proportions with these two substances and toxic symptoms which will be described below are seldom serious. The chief disadvantage is their cost, for they are both extremelv expensive.

\section{Toxic Symptoms}

The toxic symptoms of aureomycin and chloramphenicol are similar. They can be divided into three groups. In the first place we must consider those effects which are directly due to the irritative action of the antibiotics upon the gastro-intestinal tract. These are diarrhoea, nausea and vomiting. Although seldom severe enough to warrant cessation of treatment, in mild degrees they are extremely common-indeed, slight nausea and some looseness of the stools are the rule in patients receiving full dosage by the oral route. They clear up rapidly as soon as the drug is discontinued. The administration of aluminium hydroxide at the same time as the antibiotic capsules undoubtedly lessens these gastro-intestinal disturbances, but this procedure has been shown to interfere with the absorption of the antibiotic and is to be condemned. Milk may, however, be given with benefit and, if these symptoms are troublesome, it is wise to administer the drug in more frequent smaller individual doses. Secondly, there are a group of changes characterized by the appearance of ulceration in the mucous membranes of the mouth, anus and vagina. The tongue may become covered with a black coat of fur and exhibit a hairy appearance. The lesions cause much pain and soreness. The condition is very similar to that seen in some of the vitamin B deficiencies and was originally thought to be due to this cause. Such an explanation is not tenable, however, for these appearances may be seen within a few days of the administration of the antibiotic -long before any deficiency due to the malabsorption of vitamins could become clinically manifest. They are due to the action of monilia and other yeast-like organisms which flourish unopposed once the bacterial flora have been removed. Such changes have very occasionally been seen with penicillin if given orally, and are in a way a tribute to the efficacy of the antibiotic in removing most of the bacterial population from the affected areas. These symptoms may make themselves apparent for the first time after the course of the antibiotic has been completed. They are extremely unpleasant, but are not dangerous and subside as a rule in a few days without any specific treatment. Occasionally they may persist for weeks. Willcox (I95I) recently described as the ano-rectal syndrome such changes around the anus in patients with gonorrhoea treated with terramycin. Finally we have the occurrence of rare toxic and allergic manifestations, notably fever and skin rashes. A very occasional case of agranulocytosis has been described.

\section{Terramycin}

This new antibiotic, prepared from streptomyces rimosus, is very similar to aureomycin in its action, although perhaps a little less irritating to the gastro-intestinal tract. It is not yet available in this country.

\section{Polymyxin E}

The polymyxins are a group of five antibiotics produced by aerobic spore-bearing bacilli. The carlier members of the group were too toxic to be of value, but polymyxin $\mathrm{E}$ may be administered with safety. Very potent in vitro against a large number of organisms, its action in vivo is rather disappointing. Nevertheless it is likely to assume considerable importance in the treatment of urinary infections, as it is frequently the only active antibiotic available against Ps. pyocyaneus and certain strains of $\mathrm{B}$. coli and $\mathrm{B}$. proteus. It is given by intramuscular injection in daily doses of I mega unit, divided into six four-hourly injections. Administration should be continued for five or six days. It is not yet available in the country except in special circumstances.

\section{The Choice of an Antibiotic}

Theoretically it might be argued that treatment of urinary tract infections should be postponed until the organism has been identified and sen- 
sitivity tests carried out to enable the correct antibiotic to be used. Apart from the 48 hours' delay that such a procedure would generally entail, which might have serious results in fulminating infections, it is obviously quite impracticable in general practice. We believe that when dealing with acute infections it is always justifiable to commence a course of sulphonamides as soon as a clinical diagnosis is made. As by far the majority of acute infections are due to the bacillus coli, this is very likely to be attended by success. Whenever possible it is wise to obtain a specimen for bacteriological examination before treatment is started. In the male a mid-stream specimen properly collected meets the need of the bacteriologist, while in the female a catheter specimen is required.

If there is no satisfactory clinical improvement at the end of a period of 48 to 72 hours on sulphonamide therapy, the antibiotic should be changed and the choice must now depend upon the nature of the organism and the sensitivity tests if available.

Only in fulminating infections where it is felt that delay in bringing the infection under control might be attended with serious consequences is it ever justifiable to use aureomycin or chloramphenical as the initial choice of antibiotic in the absence of bacteriological indications.

In treating chronic infections the data available from the laboratory should be studied before any antibiotic therapy is instituted.

\section{Laboratory Control}

The laboratory should be in a position to furnish information as to the nature of the organism within 24 hours of receiving the specimen, and of its sensitivity to the various antibiotics in 48 hours. Occasionally if the urine is heavily infected it is possible to plate the original sample against antibiotic discs, and a rough estimation of sensitivity may thus be made at the end of a 24-hour period. The disc technique for determining sensitivity is described by Fairbrother and Martyn (1951). The dry disc can readily be prepared to a standard size and impregnated with a constant amount of the antibiotic.

\section{Resistant Strains}

Bacteria may become resistant in a few days to any of the known antibiotics. As we have seen, this phenomenon is so common when streptomycin is used that the usefulness of this otherwise excellent drug is most seriously curtailed. It is a curious fact that the chance of resistance developing with the other antibiotics depends upon the nature of the organism concerned. For example, the gonococcus is likely to develop sulphonamide resistance, while if penicillin is used the staphylo coccus aureus is by far the worst offender in this respect. There is no doubt that resistant strains do occur with both aureomycin and chlor: amphenicol, although at present it seems that these are uncommon. It must be realized, howe ever, that with wider application of these ant biotics this problem may well assume increase importance.

Furthermore, if an infection is mixed, insensitive bacteria originally present in small numbers may flourish as the sensitive organisms are removed. This fact is especially important in infections of the urinary tract and explains the not-infrequen occurrence of B. proteus or Ps. pyocyanew infection after an apparently successful course o $\bar{p}$. an antibiotic.

\section{B. Proteus and Ps. Pyocyaneus Infections}

These two organisms, together with certaio resistant strains of $B$. coli, occupy a special place in our discussion. They are most difficult to. eradicate, and testing to a full range of antibiotics is essential before the choice of treatment is made They are especially likely to occur following drainage of the bladder or kidney by a catheter and every care should be taken to avoid con $\overrightarrow{0}$ tamination. Polymyxin $\mathrm{E}$ is likely to assumexaํ important place in their treatment.

\section{Prophylactic Use of Antibiotics}

Many operations on the lower or upper urinaro tract differ from some other surgical procedures in one respect. For instance, after a prostatectom a raw surface is left. This has to granulate oves before it eventually heals with an epithelial cover ing. This raw surface is likely to become infecte

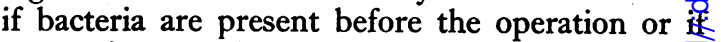
some degree of infection is introduced post operatively by urethral catheter or from the skin. via a suprapubic tube. Dukes (personal report makes an interesting and important observatioi about bladder infection from an indwelling catheter. He notices that infection occurs four of five days after a previously sterile bladder has been drained in this manner. Bacteria and pus aro found in the urine, although there may be nō clinical effects of infection. The infection arises alongside the catheter and may come on mone dramatically if the patient develops a "cathetero urethritis.' The same factors apply when a kidney

In this sphere penicillin by itself is not a reliable preventative in relation to operations. Thळ majority of gram-negative bacilli likely to cause infection of the urinary tract are penicillino resistant and may even thrive on penicillin. Thus if penicillin is used prophylactically it should bo 
combined with sulphonamides to give a wider cover. Following a prostatectomy it is usual to put the patient on 300,000 units of penicillin intramuscularly twelve-hourly and sulphatriad 2 gm. stat. followed by $\frac{1}{2} \mathrm{gm}$. six-hourly for a week. Further, if the urinary tract is infected before operation, treatment is directed to controlling the infection before any operation is undertaken. With appropriate 'cover' one seldom sees the gross sepsis which occasionally did follow bladder surgery in the pre-antibiotic days.

A short course of sulphatriad may be used prophylactically after a urethral manipulation or after a cystoscopy. Where these manipulations are repeated at regular intervals frequent courses of sulphonamides are contraindicated on account of the development of sensitization or the remote but more serious hazard of agranulocytosis.

Where uretero-colic anastomosis is contemplated the bowel is prepared with succinyl or phthalyl sulphathiazole 2 gm. six-hourly for five days before operation. Very small amounts of these are absorbed from the bowel, which is itself sterilized. Post-operatively, these cases are put on a course of sulphatriad or sulphadimidine to protect the kidneys.

Streptomycin should never be used prophylactically, and it is probably wise to keep the newer antibiotics in reserve to combat any infection that occurs in spite of the penicillin-sulphonamide cover.

\section{Cost of Antibiotics}

When arriving at a decision as to which of several antibiotics to use, the cost of treatment should always be borne in mind. Aureomycin and chloramphenicol are at present very expensive and their use should therefore be restricted to those patients where clinical considerations make it essential. It is of interest to compare the cost to a hospital of a week's treatment with the antibiotics under consideration:

-Sulphatriad-2 gm. stat., $\frac{1}{2}$ gm. 6-hourly for 7 days
Penicillin crystalline-300,000 units 12-hourly 156 Procaine penicillin-300,000 units 12-hourly I 22

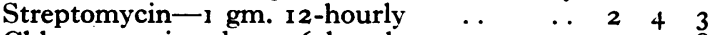

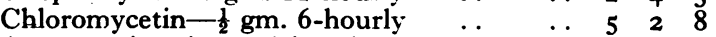

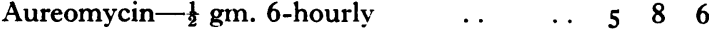

From this aspect alone it is obvious that sulphonamides are greatly to be preferred if other considerations are equal. Antibiotics have provided us with a most powerful weapon with which to combat infections of the urinary tract. Their administration, howeyer, is not without the risk of unpleasant sequelae and for their correct usage considerable clinical judgment is necessary. The closest co-operation between the bacteriologist and the clinician is essential if the best results are to be obtained.

\section{Conclusions}

I. The antibiotics are extremely valuable for the treatment of the infections of the urinary tract.

2. In acute infections a course of sulphonamides may be given while awaiting bacteriological results. Otherwise the choice of antibiotic should await the identification of the responsible organism and, if possible, its sensitivity tests. In the absence of bacteriological indications, aureomycin and chloramphenicol should only be given as the initial antibiotic in cases of grave emergency.

3. Various side-effects produced by monilia are being noticed with chloramphenicol and aureomycin.

4. The presence of underlying disease' of the urinary tract is likely to cause a relapse following treatment with the antibiotics and should be dealt with on its merits by surgical methods.

5. Penicillin and the sulphonamides are frequently used to give post-operative cover. In this respect streptomycin, chloromycetin and aureomycin are contraindicated.

6. Pyocyaneus and proteus infections occupy a a special position, in that few strains are sensitive.

\section{BIBLIOGRAPHY}

BULL, G. M., JOEKES, A. M., and LOWE, K. G. (1949), Lancet, ii, 229.

FAIRBROTHER, R. W., and MARTYN, G. (1951), F. Clin. Path., 4, 374.

FAIRBROTHER, R. W., MARTYN, G., and PARKER, L. (r95 I), Lancet, ii, 516 .

WILLCOX, R. R. (1951), Lancet, ii, 154. 


\section{CHANGE IN SUBSCRIPTION RATE}

It is much regretted that, owing to the continued rise in costs of paper and production, the price of the Postgraduate Medical Journal must now be raised-the first increase for 20 years.

On and after March I, 1952, the annual subscription rate will be $36 \mathrm{~s}$. (thirty-six shillings), post free. This rate will apply to all new subscribers from March I onwards, and to all present subscribers as their current subscriptions expire and are renewed.

\section{'CHEMOTHERAPEUTIC AND ANTIBIOTIC DRUGS' SERIES}

The Postgraduate Medical Journal's series of articles on the present status of the chemotherapeutic and antibiotic drugs commenced in August 1951. Continued monthly, in this present issue the series reaches its final article on 'The Most Recent Antibiotic Agents,' by Dr. G. Brownlee.

Chemotherapy and the Venereal Diseases

The Chemotherapy of Tropical Infections of the Bowel

The Chemotherapy of Non-Tuberculous Diseases of the Chest

The Chemotherapy of Pulmonary Tuberculosis

Chemotherapy of Non-Pulmonary Tuberculous Conditions

The Chemotherapy of Malaria

The Chemotherapy of Tuberculous Infections of the Urinary Tract

The Chemotherapy of Non-Tuberculous Infections of the Urinary Tract

The Most Recent Antibiotic Agents
R. R. Willcox, M.D.

A. R. D. Adams, M.D., F.R.C.P.

Horace Foules, M.D., F.R.C.P.

F. G. Scadding, M.D., F.R.C.P.

Peter H. Buxton, M.R.C.P.

Prof. F. Murgatroyd, M.D., F.R.C.P.

Arthur Facobs, F.R.F.P.S.

F. R. Kilpatrick, M.S., F.R.C.S.

G. Brownlee, Ph.D.', D.Sc.

For the convenience of all concerned in any way in the clinical field, the whole series is being reproduced in book form. This represents the most complete, yet compact practical information on these subjects yet published. Price $5 \mathrm{~s}$; available at all medical booksellers or direct from

The Fellowship of Postgraduate Medicine, 6o Portland Place, London, W.I

\section{ERRATUM}

In the 'Chemotherapy of Tuberculous Infections of the Urinary Tract' by Mr. F. R. Kilpatrick in" our February number, p. 84, line $\tau$, for 'sulphamerazine' read 'sulphamezathine'.

('Sulphadimidine' being the free name for 'sulphamezathine'.) 